\title{
Colorimetric Method for Determination of Amino Acids on Thin Layer and Filter Paper Chromatography Using a Modified Ninhydrin Reagent
}

\author{
Huda Shaaban Elgubbi ${ }^{1}$ and Adel Mohammad Mlitan ${ }^{2}$ \\ 1. Department of Plant, Faculty of Science, University of Misurata, Misurata 4419, Libya \\ 2. Department of Chemistry, Faculty of Science, University of Misurata, Misurata 4419, Libya
}

\begin{abstract}
A simple and rapid colorimetric method for the determination of some amino acids on thin layer chromatography (TLC) and Whatman filter paper chromatography is described. The studied compounds are cysteine, proline, methionine, tryptophan glutmine, glycine, thyrosine, threonine, leucine and lysine. The method is based on the formation of color from the reaction of stannous chloride $\left(\mathrm{SnCl}_{2}\right)$ and ninhydrin reagents. The procedure has been successfully applied to the assay of the standard amino acid preparation after TLC separation, and the results of the studied compounds were clear and favorable, comparing to the official methods. However, the improved TLC is much more efficient for the detection of amino acid. The main advantage of this method is that the detection of amino acids can be preformed visually.
\end{abstract}

Key words: Individual amino acid, modified ninhydrin reagent, $\mathrm{SnCl}_{2}$ reagent, colorimetric determination and filter paper (Whatman 14).

\section{Introduction}

The detection of amino acids is extremely important for the evaluation of protein structure and also because of their presence in various natural products. Several methods have been proposed to simplify detection of amino acids in different biological samples. The detection of amino acid on thin layer chromatograms (TLC) - one of the techniques used for visual detection and using ninhydrin reagent as a detector, was reported in several studies $[1,2]$. The reaction among amino acids, except proline, hydroxyproline, and ninhydrin, produced purple violet color [3-5]. Furthermore, its detection limits and color contrasts are not so good enough. The present communication deals with an improved reagents - stannous chloride solution $\left(\mathrm{SnCl}_{2} \cdot 2 \mathrm{H}_{2} \mathrm{O}\right)$ and ninhydrin reagent, which can give various distinguishable colors on TLC or

Corresponding author: Huda Shaaban Elgubbi, assistant professor, research field: plant eco-physiology. E-mail: hudashabban@yahoo.co.uk.
Whatman filter paper chromatography, with most of the amino acids with high sensitivities. In the present paper, the improved procedure will be described.

\section{Materials and Methods}

\subsection{Standard Preparation}

$0.1 \mathrm{~g}$ of each amino acid (aspargine, histdine, glutamine, methionine, alanine phenylalanine, tyrosine, threonine, leucine, glycine, tryptophan and proline (Sigma, USA)) was dissolved in $10 \mathrm{~mL}$ ethanol and spotted in TLC aluminum paper.

\subsection{Reagents Preparation}

\subsubsection{Reagent I}

$\mathrm{SnCl}_{2}$ solution $\left(\mathrm{SnCl}_{2} \cdot 2 \mathrm{H}_{2} \mathrm{O}\right)$ was freshly prepared: $16 \mathrm{mg}$ of $\mathrm{SnCl}_{2}$ dissolved in $10 \mathrm{~mL}(0.2 \mathrm{M})$ of citrate buffer solution with $\mathrm{pH} 5$.

Citrate buffer solution ( $\mathrm{pH}$ 5.0): $10 \mathrm{~g}$ of $\mathrm{NaOH}$ dissolved in $100 \mathrm{~mL}$ distilled water $(2 \mathrm{~N})$. 


\subsubsection{Reagent II}

Ninhydrin solution (0.31\%): $0.25 \mathrm{~g}$ of ninhydrin dissolved in $12.5 \mathrm{~mL}$ of ethanol (95\%).

\subsection{Detection on TLC Paper and Whatman Filter} Paper Chromatography

\subsubsection{Detection on TLC}

Standard amino acids solutions were spotted on the chromatography paper (Silica Gel 60 F254 TLC aluminium sheet $20 \mathrm{~cm} \times 20 \mathrm{~cm}$, thickness $0.1 \mathrm{~mm}$; Merck, Germany) by graduated capillary tubes (capacity: $5 \mu \mathrm{L}$ volume; Spectrochem). TLC papers were air-dried and subjected to TLC with butanol: glacial acetic acid:water (12:5:3, v/v) as mobile phase [6]. After development, plates were dried, sprayed with the reagent $\mathrm{I}$ and then heated at $110{ }^{\circ} \mathrm{C}$ for $6 \mathrm{~min}$ in an oven. Papers were cooled, sprayed with reagent II and then heated at $110{ }^{\circ} \mathrm{C}$ for 4-5 min. Colors were observed visually.

\subsubsection{Detection on Whatman Filter Paper} Chromatography

Standard amino acids solutions were spotted on Whatman filter paper chromatography $(20 \mathrm{~cm} \times 20$ $\mathrm{cm})$ by graduated capillary tubes. Filter papers were air-dried and subjected to TLC with butanol:glacial acetic acid:water (12:5:3, v/v) as mobile phase. After development, the filter papers were dried, sprayed with the reagent $\mathrm{I}$ and again heated at $110{ }^{\circ} \mathrm{C}$ for complete evaporation of solvent. The filter papers were then sprayed with ninhydrin (reagent II), dried in an oven at $110{ }^{\circ} \mathrm{C}$ for $6 \mathrm{~min}$, cooled and then sprayed with reagent II. Colors were always observed visually.

\section{Results and Discussion}

It has been observed that light pink color was observed in each of tested amino acids, when separated from chromatography paper (TLC aluminium), after spraying with reagent $\mathrm{I}$ with high detection limits ranging from $0.1 \mu \mathrm{g}$ to $1.0 \mu \mathrm{g}$ in hot condition [7]. But after spraying with reagent II followed by heating produce, several distinguishable color development was somewhat different almost in all the cases after second heating (Fig. 1).

Compared to the both conventional and modified TLC presented by Sinhababu [8] (Fig. 2), the improved method is clean, rapid and more sensitive in many occasions than some other suggested reagents $[8,9]$.

The same standard of amino acids (Fig. 3) were separated and detected by the proposed reagents on Whatman filter paper chromatography and under the same conditions which used in the previous experiment.

The result indicted that TLC can be used to detect amino acids. The colors of amino acids were clear, reproducible and efficient even with Whatman filter paper chromatography. There was several distinguishable color. The mechanism leading to such color formation is doubtful, but a possibility may be ascertained. One possibility is the formation of a secondary amide by the reaction between $\mathrm{SnCl}_{2}$ and amino acids, and the product obtained thus forms coloring complexes (charge transfer) with ninhydrin, colors of which are variable depending on the nature of the amino acids [10].

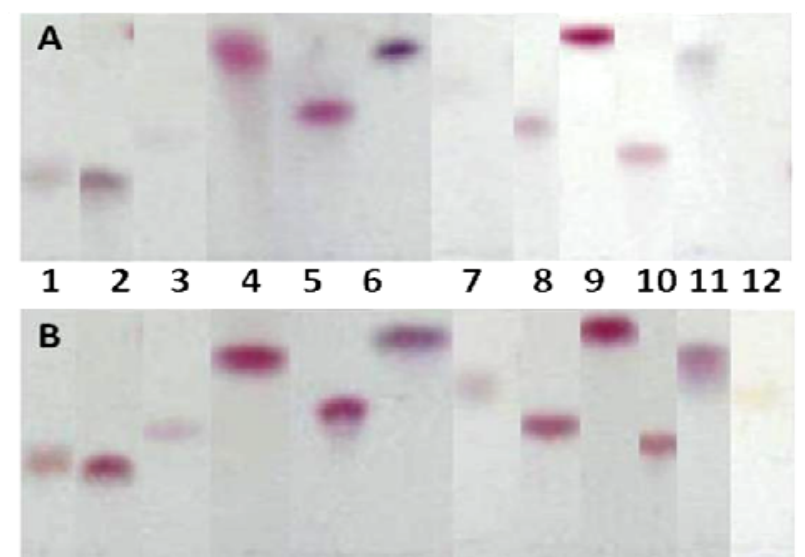

Fig. 1 The conventional (A) and modified (B) TLC chromatogram of 12 amino acids.

1-aspargine; 2-histdine 3-glutamine; 4-methionine; 5-alanine; 6-phenylalanine; 7-tyrosine; 8-threonine; 9-leucine; 10-glycine; 11-tryptophan; 12-proline. They were sprayed with $\mathrm{SnCl}_{2} \cdot 2 \mathrm{H}_{2} \mathrm{O}$ and ninhydrin reagents, on TLC with butanol:glacial acetic acid:water $(12: 5: 3, \mathrm{v} / \mathrm{v})$ as mobile phase. 


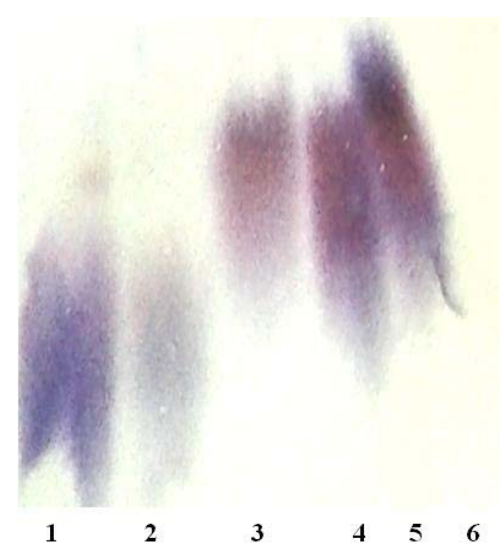

(A)

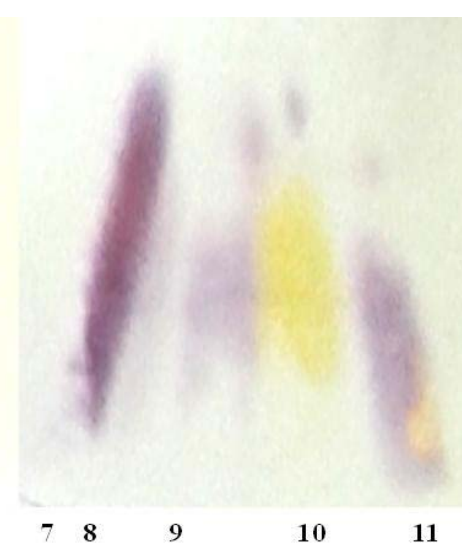

(B)

Fig. 2 The traditional TLC chromatogram (A) and the pre-staining TLC chromatogram (B) of 12 amino acids.

1-aspargine; 2-histdine; 3-glutamine; 4-methionine; 5-alanine; 6-phenylalanine; 7-tyrosine; 8-threonine; 9-leucine; 10-glycine; 11—-tryptophan; 12—proline, as shown by Reich and Schibli [11].

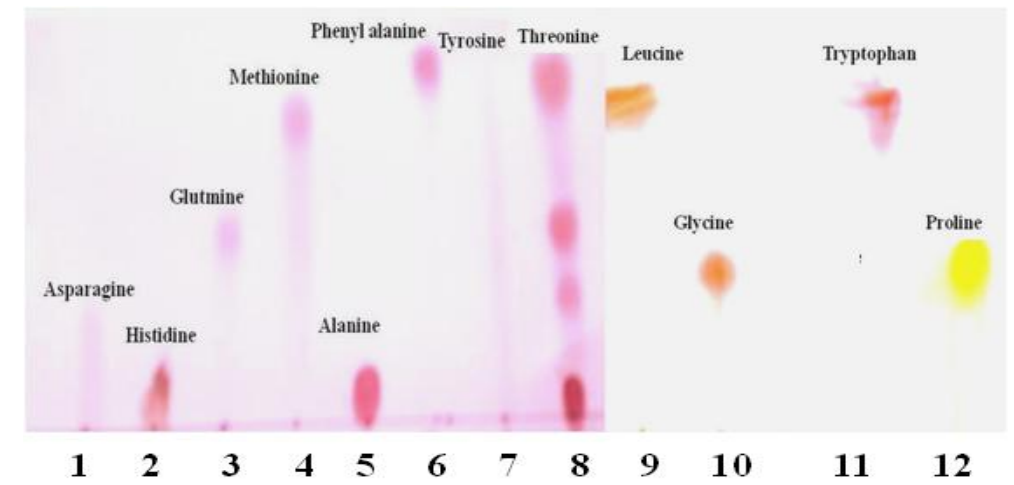

Fig. 3 Whatman filter paper chromatography of 11 amino acids.

1-alanine; 2-histdine; 3-phenylalanine; 4-methionine; 5-leucine; 6-aspargine; 7-tyrosine; 8-threonine; 9-glutamine; 10 - proline; 11 -glycine. They were sprayed with $\mathrm{SnCl}_{2} \cdot 2 \mathrm{H}_{2} \mathrm{O}$ and ninhydrin reagents, on Whatman filter paper chromatography with butanol:glacial acetic acid:water (12:5:3, v/v) as mobile phase.

The profiles of 12 amino acids from these methods were almost identical to Refs. [12-14]. Tested amino acids showed clear distinguished color (Figs. 1 and 3), and were not influenced by the modification.

Using TLC paper and Whatman filter paper in this technique will support academic research, because those materials are cheap and easy to find. On the other hand, the technique is easy to perform and the result can be predicted visually.

\section{Conclusions}

This modified method is rapid, easy and inexpensive; by the method, almost tested amino acids can easily be identified by their easily detectable colors. Moreover, the colors are stable up to $72 \mathrm{~h}$ and not effected by mild acid vapour. In addition, it is suitable for screening different amino acid and estimating protein quality. The improved method will serve the academic researchers, because it is simply to perform, effective and convenient for identification of most of the amino acids.

\section{Acknowledgments}

Authors are indebted to University of Misurata, Libya for their assistances.

\section{References}

[1] Sinhababu, A., and Basu, S. 2013. "Modified Ninhydrin Reagents to Detect Colors of Amino Acid Zones on Thin-Layer Chromatographic Plates." Journal of Planar 

Using a Modified Ninhydrin Reagent

Chromatography 26 (6): 517-20.

[2] Stahl, E. 1969. Thin Layer Chromatography: A Laboratory Handbook. New York: Springer, 873.

[3] Basak, B., Bandyopadhyay, D., Patra, M., Banerji, A., Chatterjee, A., and Banerji, J. 2005. "Role of Sulfur Compounds in the Detection of Amino Acids by Ninhydrin on TLC Plate." Journal of Chromatographic Science 43 (2): 104-5.

[4] Li, H. X., Qiu, T., Cao, Y. S., Yang, J. Y., and Huang, Z. B. 2009. "Pre-staining Paper Chromatography Method for Quantification of $\gamma$-Amino-Butyric Acid." J. Chroma. A 1216 (25): 5057-60.

[5] Sherma, E. 2000. "Thin-Layer Chromatography in Food and Agricultural Analysis." Journal of Chromatography A 880 (1-2): 129-47.

[6] Elgubbi, H. S., Mlitan, A. M., Shangi-Halfawi, A., and Zorab, A. 2014. "Phytochemical, Mineral Compounds and Anti-oxidation Studies on Pistacia lentiscus Shoot Extract." Global Journal of Medical Research B 14 (5): 33-9.

[7] Qiu, T., Li, H. X., and Cao, Y. S. 2010. "Pre-staining Thin Layer Chromatography Method for Amino Acid Detection." African Journal of Biotechnology 9 (50): 8679-81.

[8] Sinhababu, A., Basak, B., and Laskar, S. 1994. "Novel
Spray Reagent for the Identification of Amino Acids on Thin-Layer Chromatography Plates." Anal. Proc. 31 (2): 65-6.

[9] Ukil, S., Samanta, T. D., and Laskar, S. 2006. "New Reagent for Detection of Amino Acids on TLC Plates." Journal of Planar Chromatography 19: 252-4.

[10] Sinhababu, A. 2013. "Modified Ninhydrin Reagent for the Detection of Amino Acids on TLC Plates." Journal of Applied and Natural Science 5 (1): 125-7.

[11] Reich, E., and Schibli, A. 2006. High-Performance Thin-Layer Chromatography for the Analysis of Medicinal Plants. New York: Thieme Publisher, 132-56.

[12] Basak, B., Bhattacharya, U. K., and Laskar, S. 1993. "Spray Reagent for the Detection of Amino Acids on Thin-Layer Chromatography Plates." Amino Acids 4 (1-2): 193-6.

[13] Lee, S. K., Polyakova, Y., and Row, K. H. 2003. "Interrelation of Retention Factor of Amino Acids by QSPR and Linear Regression.” Bull. Korean Chem. Soc. 24 (12): 1757-62.

[14] Pataki, G., Borko, J., and Kunz, A. 1968. "The Non-destructive Detection of Amino Acids on Thin-Layer Chromatograms Using 1-Fluor-2,4-Dinitrobensene.” J. Chromatogr. 37: 499. 